\title{
Evaluation of "Energy Resonance by Cutaneous Stimulation" Among Women Treated by In Vitro Fertilization
}

\author{
Camille Gay, MD, Anne Cros, MS, Julie Berbis, MD, PhD, Florence Bretelle, MD, PhD, ${ }^{1,3}$ \\ Jeanne Perrin, MD, $\mathrm{PhD}^{1,4}$ and Blandine Courbiere, $\mathrm{MD}, \mathrm{PhD}^{1,4}$
}

\begin{abstract}
Objectives: Pregnancy rate in in vitro fertilization (IVF) depends on many factors, such as the characteristics of the couple and the clinicobiological parameters. Interest in alternative and complementary medicine (ACM) for IVF is discussed because of the lack of scientific evidence. Energy resonance by cutaneous stimulation (ERCS), an acupuncture-like technique, consists of skin stimulation to transmit vibratory messages. The aim of this study was to evaluate the effect of ERCS on live birth rates (LBRs) in IVF.

Design: A prospective observation study was performed in the Unit of Assisted Reproductive Technologies of a University Teaching Hospital. Every woman who agreed to participate in this study and received a fresh embryo transfer (ET) after IVF or Intracytoplasmic Sperm Injection (ICSI) was included. Patients randomly underwent an ERCS session on the day of ET according to the schedule of the midwife performing this technique. The control group consisted of women undergoing ET under usual conditions. The main outcome measure was the LBR per transfer.

Results: Three-hundred-eighteen women were included, 120 in the ERCS + ET group and 198 in the ET without ERCS group. None of the women dropped out. The clinical characteristics in both groups were comparable. There was a significant difference in the clinical pregnancy rates, $31.7 \%$ in the ERCS group versus $21.7 \%$ in the No ERCS group ( $p=0.037)$. The LBR in the ERCS group was nearly significantly higher, $29.2 \%$ versus $20.7 \%$ in the No ERCS group $(p=0,059)$.

Conclusions: Women undergoing ERCS on the day of ET had a significantly higher IVF pregnancy rate. However, this methodology made not possible to draw conclusions about the mechanisms that induced the increase of IVF LBRs: placebo effect, "cocooning," or ERCS self-effect? Further well-conducted studies are strongly needed to assess ERCS efficacy.
\end{abstract}

Keywords: infertility, IVF, alternative and complementary medicine, ERCS, energy resonance by cutaneous stimulation, RESC, Résonance Energétique par Stimulation Cutanée

\section{Introduction}

$\mathbf{P}$ REGNANCY RATES AFTER in vitro fertilization (IVF) depend on many prognosis factors, including features related to the couple (age, tobacco, or pesticides intoxication, drug exposition, and body mass index) and to the quality of clinicobiological care in the reproductive medicine unit. In France, the live birth rate (LBR) per cycle is $\sim 25 \% .{ }^{1}$ Despite the constant improvement in assisted reproductive technologies (ARTs) (ovarian stimulation protocols, spermatozoid selection, embryonic culture, and study of endometrial receptivity), therapeutic options remain limited after several IVF failures.

Among alternative and complementary medicine (ACM), acupuncture has gained increased interest during pregnancy

\footnotetext{
${ }^{1}$ Department of Gynecology-Obstetric and Reproductive Medicine, AP-HM, Hôpital La Conception-Hôpital Nord, Marseille, France.

${ }^{2}$ EA 3279 - Public Health, Chronic Diseases and Quality of Life - Research Unit, Aix Marseille University, Marseille, France.

${ }^{3}$ Aix Marseille University, CNRS UMR 7278, Inserm U1095, Marseille, France.

${ }^{4}$ Aix Marseille University, CNRS, IRD, Avignon University, IMBE UMR 7263, Marseille, France.
} 
and is now recommended by the French Health Authority for treatment of nausea and vomiting during early pregnancy (grade A). Several studies have shown that acupuncture could facilitate inversion of a breech fetus. ${ }^{2,3}$ In ART, researchers have shown increased interest in combining ACM and ART techniques to increase pregnancy rate; these approaches include acupuncture, ${ }^{4,5}$ auricular acupressure, ${ }^{6}$ or other Traditional Chinese Medicines, ${ }^{7}$ hypnosis, ${ }^{8}$ and relaxation. ${ }^{9}$

In a randomized study of 305 women, Qu et al. reported that the LBR was significantly higher in the group treated by auricular acupressure than in the group without acupressure or "placebo acupressure." In a case-control study of 184 couples, Levitas et al. reported a significant improvement in the implantation rate and clinical pregnancies in the group of women who underwent a hypnosis session during embryo transfer $(\mathrm{ET}){ }^{8}$ Poehl et al. observed increased pregnancy rate among patients who received psychologic support during IVF compared with those who refused support. ${ }^{10}$ However, the results of these studies on ACM are contradictory, and some studies found no improvement in pregnancy rate when comparing acupuncture with placebo. ${ }^{11,12}$ The most recent meta-analysis by Abou-Setta et al. did not find a benefit of ACM. ${ }^{13}$ Moreover, the mechanisms explaining the efficacy of these techniques have been debated at length. ${ }^{14-16}$

Energy resonance by cutaneous stimulation (ERCS) (Résonance Energétique par Stimulation Cutanée, RESC, in French) is a recent ACM technique developed in France by a physiotherapist, Patrick Fouchier. It involves creating resonance between stimulation of cutaneous points with the fingertips to perceive and transmit vibratory and wave messages in tissues. Stimulation points, inherited from Traditional Chinese Medicine, are distributed on meridians (energy circulation pathways). Stimulation points are identical in acupuncture, acupressure, and ERCS, but are stimulated in different ways, including with needles in acupuncture, firm or smooth pressure for acupressure (to reduce muscular tension), and gentle touch and intent to withdraw in the case of ERCS (to create waves between the contact points). The hypothesis of the physiologic function of ERCS is based on the production of acoustic waves (resonance) by a cutaneous point brush and transmission of theses waves in the body (fluid environment).

According to the developer (www.resc.fr), this technique would be able to sense turbulence induced by diseases and emotions to help patients calm down, whatever the disease, and should not be substituted for medical treatment. ERCS is used to treat pain based on its presumed analgesic and anxiolytic actions. There are no previous scientific publications on ERCS.

Increasing number of French midwifes and nurses are receiving training to perform ERCS, especially for pain treatment. However, no prior scientific evaluation objectively evaluated its efficacy. Given the increase in the number of French caregivers trained in ERCS with the hope of practicing this technique in hospital structures, we estimated that an evaluation with a scientific approach was necessary.

The aim of this study was to evaluate the effect of ERCS on IVF LBR, when ERCS is performed the day of ET.

\section{Materials and Methods}

A prospective observational single center study was conducted in the ART center of the University Teaching Hospital of Marseille, France, between August 1, 2015 and
April 30, 2016. After they were given information, women who received a first fresh ET after IVF and who agreed to participate were included. The exclusion criteria were as follows: oocyte or embryo donation, frozen embryo transfer, and patients who had already participated in the study after a first transfer. This study was approved by the Aix Marseille University Ethics Committee (2016-09-11-07)

Women were managed with ERCS on the day of ET on a random basis according to the schedule of the midwife who performed this technique. If the midwife was present during transfer, it was proposed that the patient could undergo this technique and participate in the study.

Prospective participants were informed of the schedule for an ERCS session, which lasted for a total of $30 \mathrm{~min}$ before and after ET and did not require invasive procedures (no needles) or clothing removal. The patient was lying down, in silence or listening relaxing music. First, the midwife asked for few questions about medical history, pains, and anxiety about IVF treatment. Then, she touched points on a meridian to feel vibratory messages. The stimulation was gentle, with fingertips, and continued until the practician can feel resonance and wave circulation between the points. The points were chosen by the midwife according to the energetic diagnosis, so as for an acupuncture procedure, in an individualized combination. The basis of the ERCS protocol included three steps: stimulation of the feet, abdomen, then head and shoulders.

On the day of ET, women were allocated to one of two groups, those who underwent an ERCS session and those who underwent ET under the usual conditions. Usually, after ET, women rested for a few minutes before they get dressed and there was no advice for bed rest. ${ }^{17}$

The couple's characteristics and clinicobiological factors involved in pregnancy rate in IVF were collected from medical records, including age, smoking status, BMI of both the man and woman, as well as the period and type of infertility (primary of secondary), ovarian reserve parameters (antiMüllerian hormone [AMH], follicular antral account, Follicle Stimulating Hormone [FSH] on day 3), origin of infertility, and sperm parameters. The following stimulation protocol data were also collected: IVF \pm Intracytoplasmic Sperm Injection (ICSI), protocol (short, long, and antagonist), total gonadotrophin dose, day of the ovulation trigger, plasmatic estradiol on the day of trigger, and rank of attempt. Then, the following biological parameters of the attempt were collected: number of oocytes collected, number of mature oocytes, number of embryos and diploid embryos, number of transferred embryos, quality of the embryo ("top" if type I or II with $<30 \%$ of fragments, in line; or "nontop"), state of development on the transfer day, and eventual technical difficulty during ET.

The main outcome measure was the LBR per transfer. The secondary outcome measures were the implantation rate (positive plasmatic human chorionic gonadotropin [hCG] 14 days after transfer), clinical pregnancy rate (embryo with heart activity at 8-week echography), early miscarriages, and ectopic pregnancies.

\section{Statistical analysis}

The LBR and secondary outcome measures were compared between patients who underwent an ERCS session on the day of ET and those who underwent transfer under the usual conditions. Qualitative variables were evaluated as the 
Table 1. Comparison of the Clinical Characteristics of Couples in the Group of Women Who Underwent an Energy Resonance by Cutaneous Stimulation Session and Those Who Did Not Undergo an Energy Resonance by Cutaneous Stimulation Session on the Day of Embryo Transfer

\begin{tabular}{|c|c|c|c|}
\hline & ERCS group $(\mathrm{n}=120)$ & No ERCS group $(\mathrm{n}=198)$ & $\mathrm{p}$ \\
\hline Woman age & $33.88( \pm 4.765)$ & $33.13( \pm 5.033)$ & 0.189 \\
\hline Woman BMI & $23.78( \pm 4.339)$ & $24.47( \pm 5.649)$ & 0.257 \\
\hline \multirow[t]{3}{*}{ Woman smoking } & Active $n=21(17.5 \%)$ & Active $n=48(24.2 \%)$ & \multirow[t]{3}{*}{0.179} \\
\hline & Former $n=21(17.5 \%)$ & Former $n=23(11.6 \%)$ & \\
\hline & Never $n=78(65.0 \%)$ & Never $n=127(64.1 \%)$ & \\
\hline \multirow[t]{2}{*}{ Infertility type Ms. (I primary, II secondary) } & I $n=64(53.3 \%)$ & I $n=115(58.1 \%)$ & \multirow[t]{2}{*}{0.408} \\
\hline & II $n=56(46.7 \%)$ & II $n=83(41.9 \%)$ & \\
\hline Poor ovarian response (Bologne criteria) (32) & $n=43(35.8 \%)$ & $n=73(36.9 \%)$ & 0.853 \\
\hline Ovulatory cause & $n=9(7.5 \%)$ & $n=17(8.6 \%)$ & 0.732 \\
\hline Tubal cause & $n=24(20.0 \%)$ & $n=38(19.2 \%)$ & 0.860 \\
\hline Idiopathic cause & $n=15(12.5 \%)$ & $n=14(7.1 \%)$ & 0.103 \\
\hline Endometriosis & $n=21(17.5 \%)$ & $n=26(13.1 \%)$ & 0.287 \\
\hline Man age & $37.69( \pm 7.406)$ & $36.69( \pm 6.748)$ & 0.229 \\
\hline Man BMI & $25.94( \pm 3.572)$ & $26.02( \pm 4.734)$ & 0.895 \\
\hline \multirow[t]{3}{*}{ Man smoking } & Active $n=36(31.3 \%)$ & Active $n=81(42.9 \%)$ & \multirow[t]{3}{*}{0.109} \\
\hline & Former $n=23(20.0 \%)$ & Former $n=27(14.3 \%)$ & \\
\hline & Never $n=56(48.7 \%)$ & Never $n=81(42.9 \%)$ & \\
\hline \multirow[t]{2}{*}{ Infertility type Mr. } & I $n=66(55.0 \%)$ & I $n=108(54.5 \%)$ & \multirow[t]{2}{*}{0.937} \\
\hline & II $n=54(45.0 \%)$ & II $n=90(45.5 \%)$ & \\
\hline OATS & $n=45(37.5 \%)$ & $n=100(50.8 \%)$ & 0.022 \\
\hline Azoospermia & $n=11(9.2 \%)$ & $n=20(10.1 \%)$ & 0.785 \\
\hline Sperm donation & $n=4(3.3 \%)$ & $n=8(4.0 \%)$ & 0.748 \\
\hline \multirow{2}{*}{ Type of couple infertility } & I $n=81(67.5 \%)$ & I $n=134(67.7 \%)$ & \multirow[t]{2}{*}{0.974} \\
\hline & II $n=39(32.5 \%)$ & II $n=64(32.3 \%)$ & \\
\hline Duration of infertility (year) & $4.689( \pm 2.74)$ & $4.449( \pm 2.65)$ & 0.544 \\
\hline
\end{tabular}

ERCS, energy resonance by cutaneous stimulation; OATS, Oligoasthenospermia.

headcount and percentage, and continuous variables were evaluated as averages with the standard deviation (or median and min max). The characteristics of both groups (ERCS and No ERCS) were compared according to conditions required, with a $\chi^{2}$ test or exact Fisher test, for qualitative values and Student's $t$ test or the Mann-Whitney test for quantitative values. Factors linked to IVF issues (LBR for the main outcome measure; plasma hCG, 8-week echography, early miscarriages, and ectopic pregnancies for secondary outcomes) were explored with adjusted models of logistic regression. The considered variables in each model were those with a significant difference in univariate analysis as well as the age of the woman (forced parameter). The results were given as the odds ratio (OR) with a confidence interval of $95 \%$. Analyses were performed with SPSS 20.0 software (SPSS, Inc., Chicago, IL). The significance level was established at $5 \%$.

\section{Results}

Three-hundred-eighteen couples were included, 120 women in the ERCS + ET group and 198 in the ET without ERCS group. No refusal of participation was recorded. None of the patients dropped out. The couple's characteristics are summarized in Tables 1 and 2. Both study populations were comparable for almost every clinicobiological parameter. More oligoasthenospermia was observed among the partners of women who did not undergo ERCS, but the necessity of ICSI was similar in both groups. Table 3 shows pregnancy issues.

After adjusting for confounding factors, significant improvement was observed at 8 weeks for pregnancies after ERCS, $31.7 \%$ in the ERCS group versus $21.7 \%$ in the No
ERCS group, $p=0.037$ (OR=1.762, CI [1.034-3.002]). The LBR was higher in the ERCS group, with a trend toward significance, $29.2 \%$ versus $20.7 \%$ in the No ERCS group, $p=0.059(\mathrm{OR}=1.698, \mathrm{CI}[0.980-2.943])$. There was a $0.5 \%$ rate of ectopic pregnancies in the ERCS group versus $0.8 \%$ in the No ERCS group, $p=0.720$, and $7.5 \%$ early miscarriages in the ERCS group versus $8.6 \%$ in the No ERCS group, $p=0.732$, according to univariate analysis. Under these conditions, multivariate analysis was not needed to conclude that there was an absence of significance for both parameters.

\section{Discussion}

After controlling for confounding factors related to pregnancy rate, it was observed that women who underwent ERCS on the day of ET had higher pregnancy rates and LBRs than women who did not undergo this technique. However, only the 8-week pregnancy rate was significantly different, whereas the LBR was not, although it trended toward significance. There was no significant difference for spontaneous early miscarriages and ectopic pregnancies. The observation that only the 8-week pregnancy rate was significantly different may be due to the lack of strength of this study.

There was a trend toward significance for the principal outcome measure concerning the pregnancy rate and LBRs (between 0.037 and 0.076). Indeed, in a small population, a few spontaneous miscarriages could be sufficient to cause variations of the $p$-value and influence significance. The number needed to treat could not be calculated before this study because no previously published study evaluated ERCS and ART, making it impossible to reasonably estimate the 
Table 2. Comparison of the Characteristics and Biological Parameters of IN Vitro Fertilization Attempts (Mean Value \pm Standard Deviation) in Women Who Did or Did Not Undergo an Energy Resonance by Cutaneous Stimulation Session on the Day of Embryo Transfer

\begin{tabular}{|c|c|c|c|}
\hline & ERCS group $(\mathrm{n}=120)$ & No ERCS group $(\mathrm{n}=198)$ & $\mathrm{p}$ \\
\hline Antral Follicular Count (AFC) & $14.1 \pm 7.65$ & $15.3 \pm 9.21$ & 0.397 \\
\hline AMH levels $(\mu \mathrm{g} / \mathrm{mL})$ & $3.01 \pm 2.31$ & $3.34( \pm 3.33)$ & 0.717 \\
\hline FSH day $3(\mathrm{UI} / \mathrm{L})$ & $7.44 \pm 2.96$ & $6.87 \pm 2.34$ & 0.056 \\
\hline Rank of the attempt & $1.89( \pm 1.11)$ & $1.66( \pm 0.90)$ & 0.124 \\
\hline \multirow[t]{2}{*}{ IVF or ICSI } & IVF $n=63(52.5 \%)$ & IVF $n=95(48.0 \%)$ & 0.435 \\
\hline & ICSI $n=57(47.5 \%)$ & ICSI $n=103(52.0 \%)$ & \\
\hline \multirow[t]{3}{*}{ Stimulation protocol } & Antagonist $n=39(32.5 \%)$ & Antagonist $n=86(43.4 \%)$ & 0.069 \\
\hline & Long-agonist $n=73(60.8 \%)$ & Long-agonist $n=94(47.5 \%)$ & \\
\hline & Short $n=8(6.7 \%)$ & Short $n=18(9.1 \%)$ & \\
\hline Total dose of gonadotrophin (UI) & $2567 \pm 1060$ & $2477 \pm 995$ & 0.583 \\
\hline Trigger day & $10.9 \pm 2.00$ & $11.0 \pm 2.21$ & 0.811 \\
\hline Plasmatic estradiol on trigger day $(\mathrm{pg} / \mathrm{mL})$ & $2548 \pm 1141$ & $2477 \pm 1202$ & 0.417 \\
\hline Type I or A endometrium, $8-13 \mathrm{~mm}$ & $n=85(70.8 \%)$ & $n=138(69.7 \%)$ & 0.830 \\
\hline Collected oocytes & $10.1 \pm 5.57$ & $9.59 \pm 5.73$ & 0.245 \\
\hline Number of mature oocytes & $7.79 \pm 4.75$ & $7.39 \pm 4.50$ & 0.509 \\
\hline Number of embryos obtained & $6.23 \pm 4.24$ & $5.83 \pm 4.03$ & 0.474 \\
\hline Number of diploid embryos obtained & $4.93 \pm 3.57$ & $4.51 \pm 2.92$ & 0.639 \\
\hline "Top" embryos & $n=39(32.5 \%)$ & $n=63(31.8 \%)$ & 0.900 \\
\hline Transferred embryo & $1.81 \pm 0.49$ & $1.74 \pm 0.47$ & 0.232 \\
\hline \multirow[t]{3}{*}{ Stage of development on the day of transfer } & $\mathrm{J} 2 n=88(73.3 \%)$ & $\mathrm{J} 2 n=151(76.3 \%)$ & 0.535 \\
\hline & $\mathrm{J} 3 n=30(25.0 \%)$ & $\mathrm{J} 3 n=46(23.2 \%)$ & \\
\hline & $\mathrm{J} 5 n=2(1.7 \%)$ & $\mathrm{J} 5 n=1(0.5 \%)$ & \\
\hline Difficulty during embryo transfer & $n=4(3.3 \%)$ & $n=7(3.5 \%)$ & 0.924 \\
\hline
\end{tabular}

AMH, anti-Müllerian hormone; IVF, in vitro fertilization; FSH, Follicle Stimulating Hormone; ICSI, Intracytoplasmic Sperm Injection.

expected benefit on the LBR. Biases should be considered in this study and the results. This was a prospective casecontrol study and not a randomized controlled study. The allocation of women in each group was random, but not randomized, because the study depended on whether the midwife was available to perform ERCS sessions. Physicians who scheduled the date of transfer (on trigger day) were unaware of the midwife's schedule; therefore, they did not know whether she would be present on the day of transfer, which limited the randomization procedure.

This was not a blinded study because women knew whether they underwent an ERCS session and physicians knew on the day of transfer whether the midwife was available to perform ERCS sessions. However, a double blinded randomized study for ERCS evaluation is difficult to establish because the caregiver knows always what technique he is doing. In this study, only senior physicians were allowed to perform ET, and they had a similar pregnancy rate (unpublished data but necessary quality criteria for centers). Moreover, the midwife's schedule was not predictable, excluding the bias linked to the physician. The study did not assess whether women used other ACM techniques in addition of ERCS during the IVF attempt (acupuncture, relaxation, etc.), and these techniques are commonly used by infertile women. No previous international study has published data on ERCS, and there are no prior studies concerning the potential mechanism of the efficiency of ERCS. In this study, the mechanisms leading to higher pregnancy rates could be ERCS self-effect, placebo effect, or cocooning (increased therapeutic attention and caring, in a comfortable environment).

Data on acupuncture are more widespread. Several studies have shown an interest in ACM in supporting ART, but meta-analyses are contradictory. Shen et al. ${ }^{5}$ did not find a significant difference in clinical pregnancy rate between groups with acupuncture versus without acupuncture during ET. They only found significant differences when acupuncture was performed $30 \mathrm{~min}$ before transfer, during the implantation period, and $25 \mathrm{~min}$ before and after ET. Several

Table 3. Pregnancy Outcome After In Vitro Fertilization in the Energy Resonance by Cutaneous Stimulation and No Energy Resonance by Cutaneous Stimulation Groups

\begin{tabular}{|c|c|c|c|c|}
\hline & $\begin{array}{c}\text { ERCS } \\
\text { group } \mathrm{n}=120\end{array}$ & $\begin{array}{c}\text { No ERCS } \\
\text { group } \mathrm{n}=198\end{array}$ & $\begin{array}{l}\text { Univariate } \\
\text { analysis } \mathrm{p}\end{array}$ & Multivariate analysis $\mathrm{p}$ \\
\hline Positive hCG (\%) & $n=48(40.0)$ & $n=62(31.3)$ & 0.114 & $\begin{array}{c}p=0.076 \\
\mathrm{OR}=1.562 \text { CI }[0.954-2.556]\end{array}$ \\
\hline $\begin{array}{l}\text { Ongoing pregnancy } \\
\text { at } 8 \text { weeks }(\%)\end{array}$ & $n=38(31.7)$ & $n=43(21.7)$ & 0.048 & $\begin{array}{c}p=0.037 \\
\mathrm{OR}=1.762 \text { CI [1.034-3.002] }\end{array}$ \\
\hline Live birth $(\%)$ & $n=35(29.2)$ & $n=41(20.7)$ & 0.086 & $\begin{array}{c}p=0.059 \\
\mathrm{OR}=1.698, \text { CI }[0.980-2.943]\end{array}$ \\
\hline
\end{tabular}

CI, confidence interval; ERCS, energy resonance by cutaneous stimulation; hCG, human chorionic gonadotropin; OR, odds ratio. 
sessions of acupuncture or sessions at specific moments during the attempt could be necessary to improve pregnancy rates. In their meta-analysis, Manheimer et al. concluded that there was not a significant improvement of the IVF pregnancy rates after one to three acupuncture sessions, ${ }^{18,19}$ except in a subgroup of studies with low pregnancy rates $\left(<32 \%\right.$, close to the European mean results $\left.{ }^{20}\right)$, which had significantly higher pregnancy rate. After excluding articles using sham acupuncture (called the Streiberger technique), Zheng et al. ${ }^{21}$ found a significant improvement in the clinical pregnancy rate and LBR whenever the session was performed. Sham needling technique uses blunt needles covered by plaster, which gives the impression that the needle is inserted into the skin. The authors suggest that this control technique, to protect patient blindness in the study, could help with this type of study. The needle tip, when touching the skin, stimulates acupuncture points and is thus not inert. Furthermore, analysis of only a subgroup of studies using the Streitberger technique as a control showed a decrease in LBR in the acupuncture group. These results may appear to be unbelievable considering that no secondary effect of acupuncture on pregnancy has ever been demonstrated.

Anderson and Rosenthal proposed several hypotheses to explain the differences between the results of the different meta-analyses and the difficulty to inspire light acupuncture interest: (1) the placebo is not inert and should not be compared with acupuncture, instead, it should be compared with therapeutic abstention; (2) acupuncture cannot exactly follow a protocol without being adapted to the patient and clinical context, as recommended by Traditional Chinese Medicine; and (3) the use of different acupuncture protocols makes it difficult to build methodology and interpret metaanalyses. $^{22}$ Recently, in a meta-analysis of 30 studies, Qian et al. found an improvement in pregnancy rate in the acupuncture group. ${ }^{23}$ Moreover, the authors reported heterogeneity in the results, according to the treated population; acupuncture seemed to be more efficient in an Asian population with electroacupuncture or when sessions were performed during ovarian stimulation (rather than during ET). The limitations of the meta-analysis were the differences in the acupuncture protocols and in the number of transferred embryos. Among a population of women with polycystic ovarian syndrome, Jo and $\mathrm{Lee}^{24}$ showed in a meta-analysis an increase in pregnancy rate and a decrease in ovarian hyperstimulation risk when patients underwent acupuncture, but they failed to show a significant increase in LBR. These meta-analyses do not reach the same conclusions because of the heterogeneity in the methodologies and inclusion criteria of studies. Unlike the acupuncture protocol for nausea treatment during pregnancy (P6 acupuncture point) or inversion of a breech fetus (BL67 point), most studies do not use a standardized procedure for acupuncture. Thus, the studies concerning IVF do not use precise stimulation points, a standardized number of sessions, or an optimal moment when performing sessions. However, in 2012, a collaboration of 28 international acupuncturists working on fertility agreed to describe an acupuncture protocol for infertility. ${ }^{25}$ To the best of the authors' knowledge, no previously published study has been performed with this protocol. The authors underlined the importance of adapting the protocol to the patient and clinical context, explaining the limits of its utilization.
Available studies make differences in the type of acupuncture (manual, moxibustion, and electroacupuncture), use of a control group (with or without placebo), and type of placebo, among other factors. Standardization of these techniques seems to be essential to compare studies and conduct a reliable meta-analysis.

Regarding the explanation of the biological function or physiology of acupuncture, studies suggest that acupuncture could reduce stress during ET and improve pregnancy rate. ${ }^{26}$ Acupuncture would be able to improve blood flow and decrease uterine artery impedance. ${ }^{27,28}$ Some studies suggested that acupuncture is able to regulate growth factor rates in follicular fluid and blood as well as improve oocyte quality. ${ }^{29}$ Likewise, there is no consensus on this subject.

Concerning ERCS, it would have been interesting to add an evaluation of the well-being and stress induced by IVF in this study, which would have allowed for an analysis of the potential beneficial effects on stress and exploration of a physiopathologic hypothesis of ERCS mechanism of action. Stress could indeed be involved in ART failures. ${ }^{30}$

Manheimer et al. reported that the use of "sham acupuncture" as a control group is not justified because, even if acupuncture increases the LBR only because of a psychosomatic effect (like stress reduction), this effect could be a part of the function of acupuncture and cannot be compared with another stress reduction method. ${ }^{31}$ Moreover, even if the improvement in the pregnancy rate is only due to the placebo effect, it could be interesting to use these techniques, even though many mechanisms for embryonic implantation remain unexplained. Concerning the safety of acupuncture, if beneficial effects remain unproven, no serious or frequent adverse effects have been reported with ACM. ${ }^{32,33}$

\section{Conclusions}

Despite biases which are difficult to avoid in studies on ACM, it was shown that women undergoing ERCS on the day of ET had significantly higher pregnancy rate and a trend to higher LBR. ACM must be studied with a thorough methodology to differentiate the effect of the own ACM technique and the placebo effect (due to cocooning). A blinded, randomized study, on a larger population comparing ERCS with therapeutic abstention and with a group receiving "sham ERCS" (relaxation session with fingertips on fictive points, with a caregiver untrained in ERCS) is needed. This would be the only methodology that could help to distinguish the effect of ERCS from the "cocooning" effect. In this case, the study would be single blinded, with the patient not knowing whether she is receiving true or sham ERCS. However, even if ERCS had a placebo effect or cocooning effect, the improvement in the pregnancy rate would be a sufficient argument to encourage supporting ACM as a complement to ART.

\section{Acknowledgment}

The project leading to this publication has received funding from Excellence Initiative of Aix-Marseille UniversityA*MIDEX, a French “Investissements d'Avenir" programme. (No. ANR-11-IDEX-301-02).

\section{Author Disclosure Statement}

No competing financial interests exist. 


\section{References}

1. Nelson SM, Lawlor DA. Predicting live birth, preterm delivery, and low birth weight in infants born from in vitro fertilisation: A prospective study of 144,018 treatment cycles. PLoS Med 2011;8:e1000386.

2. Guittier M-J, Pichon M, Dong H, et al. Moxibustion for breech version: A randomized controlled trial. Obstet Gynecol 2009;114:1034-1040.

3. Vas J, Aranda JM, Nishishinya B, et al. Correction of nonvertex presentation with moxibustion: A systematic review and metaanalysis. Am J Obstet Gynecol 2009;201:241-259.

4. di Villahermosa DIM, dos Santos LG, Nogueira MB, et al. Influence of acupuncture on the outcomes of in vitro fertilisation when embryo implantation has failed: A prospective randomised controlled clinical trial. Acupunct Med 2013;31:157-161.

5. Shen $\mathrm{C}$, Wu M, Shu D, et al. The role of acupuncture in in vitro fertilization: A systematic review and metaanalysis. Gynecol Obstet Invest 2015;79:1-12.

6. Qu F, Zhang D, Chen L-T, et al. Auricular acupressure reduces anxiety levels and improves outcomes of in vitro fertilization: A prospective, randomized and controlled study. Sci Rep 4:5028.

7. Hullender Rubin LE, Opsahl MS, Wiemer KE, et al. Impact of whole systems traditional Chinese medicine on in-vitro fertilization outcomes. Reprod Biomed Online 2015;30:602-612.

8. Levitas E, Parmet A, Lunenfeld E, et al. Impact of hypnosis during embryo transfer on the outcome of in vitro fertilization-embryo transfer: A case-control study. Fertil Steril 2006;85:1404-1408.

9. Okhowat J, Murtinger M, Schuff M, et al. Massage therapy improves in vitro fertilization outcome in patients undergoing blastocyst transfer in a cryo-cycle. Altern Ther Health Med 2015;21:16-22.

10. Poehl M, Bichler K, Wicke V, et al. Psychotherapeutic counseling and pregnancy rates in in vitro fertilization. $\mathbf{J}$ Assist Reprod Genet 1999;16:302-305.

11. Andersen D, Løssl K, Nyboe Andersen A, et al. Acupuncture on the day of embryo transfer: A randomized controlled trial of 635 patients. Reprod Biomed Online 2010;21:366-372.

12. Craig LB, Rubin LEH, Peck JD, et al. Acupuncture performed before and after embryo transfer: A randomized controlled trial. J Reprod Med 2014;59:313-320.

13. Abou-Setta AM, Peters LR, D'Angelo A, et al. Post-embryo transfer interventions for assisted reproduction technology cycles. Cochrane Database Syst Rev 2014;8:CD006567

14. Yang B-Z, Cui W, Li J. Effects of electroacupuncture intervention on changes of quality of ovum and pregnancy out-come in patients with polycystic ovarian syndrome [In Chinese]. Zhen Ci Yan Jiu 2015;40:151-156.

15. Yan H, Huang X-H, Deng G-F. Observation on therapeutic effect of acupuncture and moxibustion on disorders of myometrial gland [In Chinese]. Zhongguo Zhen Jiu 2008; 28:579-581.

16. Shuai Z, Lian F, Li P, Yang W. Effect of transcutaneous electrical acupuncture point stimulation on endometrial receptivity in women undergoing frozen-thawed embryo transfer: A single-blind prospective randomised controlled trial. Acupunct Med 2015;33:9-15.

17. Craciunas L, Tsampras N. Bed rest following embryo transfer might negatively affect the outcome of IVF/ICSI: A systematic review and meta-analysis. Hum Fertil (Camb) 2016;19:16-22.

18. Manheimer E, Zhang G, Udoff L, et al. Effects of acupuncture on rates of pregnancy and live birth among wo- men undergoing in vitro fertilisation: Systematic review and meta-analysis. BMJ 2008;336:545-549.

19. Manheimer E, van der Windt D, Cheng K, et al. The effects of acupuncture on rates of clinical pregnancy among women undergoing in vitro fertilization: A systematic review and meta-analysis. Hum Reprod Update 2013;19:696-713.

20. de Mouzon J, Goossens V, Bhattacharya S, et al. Assisted reproductive technology in Europe, 2007: Results generated from European registers by ESHRE. Hum Reprod 2012;27:954-966.

21. Zheng $\mathrm{CH}$, Huang GY, Zhang MM, Wang W. Effects of acupuncture on pregnancy rates in women undergoing in vitro fertilization: A systematic review and metaanalysis. Fertil Steril 2012;97:599-611.

22. Anderson B, Rosenthal L. Acupuncture and in vitro fertilization: Critique of the evidence and application to clinical practice. Complement Ther Clin Pract 2013;19:1-5.

23. Qian Y, Xia X-R, Ochin H, et al. Therapeutic effect of acupuncture on the outcomes of in vitro fertilization: A systematic review and meta-analysis. Arch Gynecol Obstet 2017;295:543-558.

24. Jo J, Lee YJ. Effectiveness of acupuncture in women with polycystic ovarian syndrome undergoing in vitro fertilisation or intracytoplasmic sperm injection: A systematic review and meta-analysis. Acupunct Med 2017;35:162-170.

25. Smith CA, Grant S, Lyttleton J, Cochrane S. Using a Delphi consensus process to develop an acupuncture treatment protocol by consensus for women undergoing Assisted Reproductive Technology (ART) treatment. BMC Complement Altern Med 2012;12:88.

26. Balk J, Catov J, Horn B, et al. The relationship between perceived stress, acupuncture, and pregnancy rates among IVF patients: A pilot study. Complement Ther Clin Pract 2010;16:154-157.

27. Huang D, Huang G, Lu F, et al. Acupuncture for infertility: Is it an effective therapy? Chin J Integr Med 2011;17:386-395.

28. Cakmak YO. Electroacupuncture reduces uterine artery blood flow impedance. Taiwan J Obstet Gynecol 2010;49:390.

29. Chen J, Liu L, Cui W, Sun W. [Effects of electroacupuncture on in vitro fertilization-embryo transfer (IVF-ET) of patients with poor ovarian response]. Zhongguo Zhen Jiu 2009;29:775-779.

30. Smith CA, Ussher JM, Perz J, et al. The effect of acupuncture on psychosocial outcomes for women experiencing infertility: A pilot randomized controlled trial. J Altern Complement Med 2011;17:923-930.

31. Manheimer E. Selecting a control for in vitro fertilization and acupuncture randomized controlled trials (RCTs): How sham controls may unnecessarily complicate the RCT evidence base. Fertil Steril 2011;95:2456-2461.

32. Clarkson CE, O'mahony D, Jones DE. Adverse event reporting in studies of penetrating acupuncture during pregnancy: A systematic review. Acta Obstet Gynecol Scand 2015;94:453-464.

33. Carr DJ. The safety of obstetric acupuncture: Forbidden points revisited. Acupunct Med 2015;33:413-419.

Address correspondence to: Blandine Courbiere, $M D, P h D$ Unit of Assisted Reproductive Technologies Department of Gynecology-Obstetric AP-HM La Conception 147 bd Baille Marseille 13005 France

E-mail: blandine.courbiere@univ-amu.fr 